\title{
Modulation of the gut microbiome: a systematic review of the effect of bariatric surgery
}

\author{
Yan Guo ${ }^{1, *}$, Zhi-Ping Huang ${ }^{2,3, *}$, Chao-Qian Liü,*, Lin Qi', Yuan Sheng ${ }^{3}$ and \\ Da-Jin Zou' ${ }^{1}$
}

1Department of Endocrinology, Changhai Hospital, Shanghai, China, ${ }^{2}$ Third Department of Hepatic Surgery, Shanghai Eastern Hepatobiliary Surgery Hospital, Shanghai, China, ${ }^{3}$ Department of General Surgery, Shangai Changhai Hospital, Shanghai, China, and ${ }^{4}$ Department of Orthopaedics, the Second Xiangya Hospital, Central South University, Changsha, Hunan, China

*(Y Guo, Z-P Hunag and C-Q Liu contributed equally to this work)
Correspondence should be addressed to Y Sheng or D-J Zou Email shengyuan.smmu@aliyun. com or zoudajin@hotmail. com

\section{Abstract}

Objective: Bariatric surgery is recommended for patients with obesity and type 2 diabetes. Recent evidence suggested a strong connection between gut microbiota and bariatric surgery.

Design: Systematic review.

Methods: The PubMed and OVID EMBASE were used, and articles concerning bariatric surgery and gut microbiota were screened. The main outcome measures were alterations of gut microbiota after bariatric surgery and correlations between gut microbiota and host metabolism. We applied the system of evidence level to evaluate the alteration of microbiota. Modulation of short-chain fatty acid and gut genetic content was also investigated.

Results: Totally 12 animal experiments and 9 clinical studies were included. Based on strong evidence, 4 phyla (Bacteroidetes, Fusobacteria, Verrucomicrobia and Proteobacteria) increased after surgery; within the phylum Firmicutes, Lactobacillales and Enterococcus increased; and within the phylum Proteobacteria, Gammaproteobacteria, Enterobacteriales Enterobacteriaceae and several genera and species increased. Decreased microbial groups were Firmicutes, Clostridiales, Clostridiaceae, Blautia and Dorea. However, the change in microbial diversity is still under debate. Faecalibacterium prausnitzii, Lactobacillus and Coprococcus comes are implicated in many of the outcomes, including body composition and glucose homeostasis.

Conclusions: There is strong evidence to support a considerable alteration of the gut microbiome after bariatric surgery. Deeper investigations are required to confirm the mechanisms that link the gut microbiome and metabolic alterations in human metabolism.

\section{Introduction}

Obesity, combined with its related comorbidities, such as type 2 diabetes (T2D), cardiovascular disease and psychological disorders, is attracting increasing attention and becoming a worldwide health problem. Although additional studies are needed to further demonstrate mechanistic basis of these benefits, there is clinical evidence that show the long-term benefit of bariatric
() 2018 European Society of Endocrinology Printed in Great Britain
European Journal of

Endocrinology

(2018) 178, 43-56 surgery (1), supporting the inclusion of bariatric surgery among the anti-diabetes interventions for people with type 2 diabetes and obesity (2). The gut microbiome has been recognized as a potential contributor for regulating host metabolism in patients undergoing bariatric surgery $(3,4)$. Furthermore, recent research found a great similarity between the genetic content of the human and rat gut 
(5), suggesting that more investigations on gut microbiota from both human and rodents are needed.

Bariatric surgery, particularly Roux-en-Y gastric bypass (RYGB), induces important changes in the digestive tract, including decreased acid production and anatomical gut rearrangement, which may affect the modification of the gut microbiota. Given the impact that the gut microbiota has on host metabolism through several mechanisms (6), it is probably that this complex enteric microbial community contributes to surgery-mediated weight loss and the maintenance of the benefit for host metabolism post-surgery.

Magouliotis et al. composed a meta-analysis to report the impact of bariatric surgery on metabolic and gut microbiota profiles recently (7); however, the alteration of gut microbiota and the host-microbiota crosstalk on patients undergoing bariatric surgery still warrants investigation due to the lack of a quantitative summary. So we composed this systematic review to further identify the alteration of gut microbial composition after bariatric surgery and gain a better understanding of the interaction between the host metabolism and the microbiome $(2,8)$.

\section{Subjects and methods}

\section{Data sources}

The preferred reporting items for systematic reviews and meta-analysis (PRISMA) guidelines were used to guide the search (9). We retrieved studies from PubMed and OVID Embase. The date of the last search was December 2016. In addition, we searched the reference list of the studies we retrieved. The main search terms were 'Bariatric Surgery', 'Sleeve gastrectomy', 'Gastrojejunostomy', 'Gastrojejunostomies', 'duodenal-jejunal bypass', 'DJB', 'RYGB', 'Gastrointestinal Microbiome', 'flora' and 'microbial'.

\section{Study selection}

The inclusion criteria were as follows:

(a) clinical trials or observational experiments reporting primary data of bariatric surgery;

(b) alteration of the gut microbiota profile after bariatric surgery was used as an outcome measure;

(c) composed in English.
We applied the exclusion criteria as follows:

(d) letters to the editor, conference proceedings, abstracts or review;

(e) the study included the patients combined with certain diseases, such as Crohn's disease, ulcerative colitis, gastric tumors or lower gastrointestinal tumors.

\section{Data extraction and quality assessment}

Two investigators independently reviewed the selected studies and extracted data from them. To assess the quality of the human trials, we used the guideline called 'The Newcastle-Ottawa Scale (NOS) for cohort studies' $(10,11)$, which contains 8 items based on 3 sections of the article regarding selection, comparability and outcome. We also evaluated the included animal reports with The Animal Research: Reporting of the In Vivo Experiments (ARRIVE) guideline, which refers to several parts of the manuscript: title, abstract, introduction, method, results and discussion (12).

Importantly, we considered an animal research to be of good quality if the score was more than 14 and a clinical study to be of good quality if the score was more than 7. Otherwise, the study was labeled 'poor quality'. The third assessor was needed if there was disagreement between the two investigators.

\section{Data synthesis and level of evidence}

Considering the heterogeneity of the study methodology, a quantitative analysis of the system of evidence level was performed (Table 1) $(13,14,15,16)$. We evaluated the evidence of the alteration of each specific taxon of microbiota by strictly considering the number, the methodological quality and the consistency of studies manifesting this alteration. Findings were considered consistent when more than $75 \%$ of the studies that reported the specific microbiota showed the same trend of alteration (Table 1).

\section{Results}

\section{Studies included and methodological quality}

Initially, 477 reports were recovered during the retrieval and a total of 22 papers reporting 21 studies, including 9 human studies and 12 animal experiments, were included 
Table 1 Levels of evidence.

Strong

Moderate

Weak

Inconclusive
Consistent findings ( $\geq 75 \%)$ in at least 2 high-quality studies

Consistent findings $(\geq 75 \%)$ in one high-quality study and at least one low-quality study

Findings of one high-quality study or consistent findings ( $\geq 75 \%)$ in at least 3 or more low-quality studies Inconsistent findings, or less than 3 low-quality studies available in this review (Fig. 1). In total, 6 types of bariatric surgeries were applied: RYGB, vertical banded gastroplasty (VBG), sleeve gastroplasty (SG), duodenal-jejunal bypass (DJB), bilio-intestinal bypass (BIB) and ileal interposition (IT). Using the above-described criteria to define the quality, of the 21 overall studies, we found that 6 animal studies and 5 clinical studies were of good quality (Tables 2, 3 and 4).

\section{Altered microbial composition}

The results of the population of the microbiota represent new findings based on the level of evidence (Table 5 and Supplementary Table 1, see section on supplementary data given at the end of this article), and microbial species analyzed in only one study were omitted.

\section{Microbial richness and diversity}

The change in the richness and diversity of microbiota after surgery was still controversial. The gut microbiota richness, as estimated by Chao1 (17) and abundance-

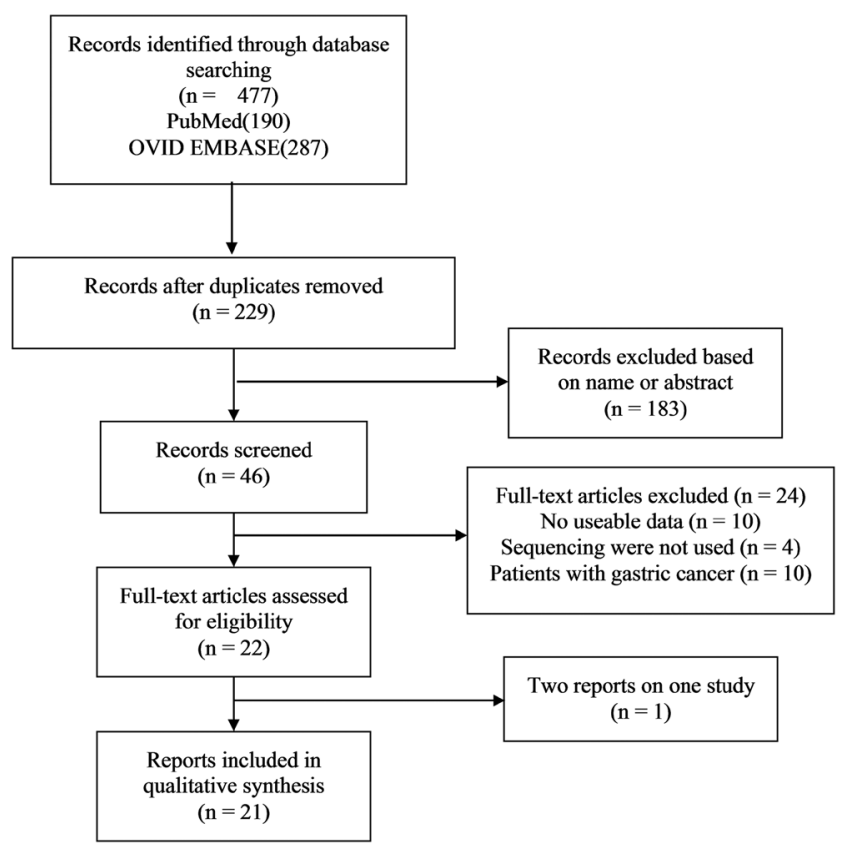

Figure 1

Flow chart of study selection. based coverage estimators, increased after RYGB in a human study (18) but showed no difference in an animal model (19). For patients undergoing BIB, there was a significant decrease in the mean microbial richness estimated by Chao1 in post-surgery subjects (20). Murphy et al. demonstrated that the mean number of species increased 1 year after surgery (21). Another study showed that species richness was higher 3 months after RYGB and that this higher richness was maintained at 1 year (22). However, the gut microbiota diversity, as estimated by the Shannon index, was reported to be higher after surgery both in animal studies $(19,23)$ and in people with obesity undergoing RYGB (22) and BIB (20). However, a human study in 2014 (24) showed a similar Shannon diversity index before and after RYGB. In 2016, it was shown that the Shannon diversity index of the gut microbiota of the RYGB group remained significantly depressed 3 weeks postoperatively (25).

\section{Microbiota altered on strong evidence}

The population of 21 microbial taxa was altered on a strong level of evidence (Table 5 and Supplementary Table 1). Metagenomic sequencing of the human gut microbiome before and after RYGB in diabetic patients exhibited an increased population of Proteobacteria $(22,26)$, resembling the results observed in a study focusing on patients undergoing BIB surgery (20). An increased population of Proteobacteria was observed in diet-induced mice with obesity after RYGB (27). Experimental results on rats showed an increase of Proteobacteria after DJB (28) and RYGB $(19,25)$. In addition, Li et al. found substantial shifts of the main gut phyla toward higher concentrations of Proteobacteria, specifically Enterobacter hormaechei (E. hormaechei), from a non-obese rat model characterizing the effect of RYGB (29). An increase in E. hormaechei was also observed in patients undergoing either RYGB or VBG (30). An upregulation of the abundance of Bacteroidetes was observed on animal experiences after $\operatorname{RYGB}(19,27)$ and SG (31). However, contrary results were provided on rats after DJB (28). Two studies reported the increase of the phylum Fusobacteria in $2016(19,22)$. The abundance of phylum Verrucomicrobia was found to be increased in patients after RYGB (24) and mice after RYGB (27) and DJB (32). 
Table 2 Methodological assessment for animal experiments.

\begin{tabular}{|c|c|c|c|c|c|c|c|c|c|c|c|c|c|c|c|c|c|c|c|c|c|}
\hline \multirow[b]{2}{*}{ Study } & \multicolumn{21}{|c|}{ Number of items of ARRIVE* checklist } \\
\hline & 1 & 2 & 3 & 4 & 5 & 6 & 7 & 8 & 9 & 10 & 11 & 12 & 13 & 14 & 15 & 16 & 17 & 18 & 19 & 20 & Score \\
\hline (63) & 1 & 1 & 1 & 1 & 0 & 1 & 1 & 1 & 1 & 1 & 0 & 1 & 1 & 1 & 1 & 1 & 0 & 1 & 1 & 0 & 16 \\
\hline (25) & 1 & 1 & 1 & 1 & 1 & 1 & 1 & 1 & 1 & 1 & 1 & 1 & 1 & 0 & 1 & 0 & 0 & 1 & 1 & 0 & 16 \\
\hline (19) & 1 & 1 & 1 & 1 & 0 & 1 & 1 & 0 & 1 & 0 & 1 & 1 & 1 & 0 & 1 & 1 & 1 & 1 & 1 & 1 & 16 \\
\hline (27) & 1 & 1 & 1 & 1 & 0 & 0 & 1 & 1 & 0 & 0 & 1 & 1 & 1 & 0 & 1 & 1 & 1 & 1 & 1 & 1 & 15 \\
\hline (29) & 1 & 1 & 1 & 1 & 1 & 1 & 1 & 0 & 1 & 0 & 0 & 1 & 0 & 0 & 1 & 1 & 1 & 1 & 1 & 1 & 15 \\
\hline (32) & 1 & 1 & 1 & 1 & 1 & 1 & 1 & 0 & 1 & 0 & 1 & 1 & 1 & 1 & 1 & 1 & 0 & 0 & 1 & 0 & 15 \\
\hline (23) & 1 & 1 & 0 & 1 & 1 & 1 & 1 & 0 & 1 & 0 & 1 & 1 & 1 & 0 & 1 & 0 & 0 & 1 & 1 & 1 & 14 \\
\hline (28) & 1 & 1 & 0 & 1 & 0 & 0 & 1 & 1 & 1 & 0 & 0 & 1 & 1 & 1 & 1 & 1 & 1 & 1 & 0 & 1 & 14 \\
\hline (31) & 1 & 1 & 1 & 1 & 0 & 1 & 0 & 0 & 0 & 1 & 1 & 1 & 1 & 1 & 1 & 1 & 0 & 1 & 1 & 0 & 14 \\
\hline (37) & 1 & 1 & 0 & 1 & 1 & 1 & 1 & 0 & 0 & 1 & 0 & 1 & 1 & 0 & 1 & 0 & 1 & 1 & 0 & 1 & 13 \\
\hline (33) & 1 & 1 & 1 & 0 & 0 & 1 & 0 & 0 & 0 & 1 & 1 & 1 & 1 & 0 & 1 & 1 & 1 & 1 & 1 & 0 & 13 \\
\hline (34) & 1 & 1 & 1 & 1 & 0 & 0 & 0 & 0 & 0 & 0 & 0 & 1 & 0 & 0 & 1 & 1 & 0 & 1 & 1 & 1 & 10 \\
\hline
\end{tabular}

*ARRIVE, reporting of the in vivo experiments.

The population of Gammaproteobacteria increased in rats after RYGB $(19,25,29)$, DJB (33), SG (23) and IT surgery (34). The fecal samples from patients undergoing RYGB or BIB also implies a significant increase of Gammaproteobacteria $(20,35)$. Lactobacillales was found to increase after bariatric surgeries $(20,25)$. The results from an experiment using rat models undergoing RYGB and a clinic cohort investigating BIB demonstrated an increase in the population of Enterobacteriales $(20,27)$. The family of Enterobacteriaceae showed an increase in quantity according to the pyrosequencing analysis of fecal sample from a non-obese rat model (35), three people with obesity after gastric bypass (29) and eleven people with obesity undergoing BIB (20). Both animal and human studies indicated an upregulation of the concentration of Alistipes after gastric bypass $(18,19,27)$. Postoperative increases in the population of Escherichia were found in diabetic IT-operated rats and mice after RYGB $(27,34)$, and further clinical investigations focusing on RYGB revealed the same trend of the alteration of Escherichia $(18,30)$. The increase of Enterococcus was observed on humans and rats $(19,30)$. Additionally, the population of

Table 3 Methodological assessment for human studies.

\begin{tabular}{|c|c|c|c|}
\hline \multirow[t]{2}{*}{ Study } & \multicolumn{3}{|c|}{ Newcastle-Ottawa scale for cohort studies } \\
\hline & Selection & Comparability & Outcome \\
\hline (30) & 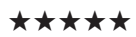 & $\star \star$ & $\star \star \star \star$ \\
\hline (20) & $\star \star \star \star \star$ & 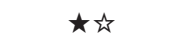 & $\star \star \star \star$ \\
\hline (22) & $\star \star \star \star \star$ & $\star \Delta$ & $\star \star \star \star$ \\
\hline (21) & $\star \star \star \star$ & $\star$ 公 & $\star \star \star$ \\
\hline (18), (38) & $\star \star \star \star$ & 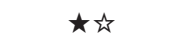 & $\star \star \star \star$ \\
\hline (26) & $\star \star \star \star ~$ & $\star \Delta$ & $\star \star \star え$ \\
\hline (36) & 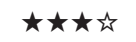 & 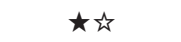 & $\star \star \star \star$ \\
\hline (24) & $\star \star \star \star 々$ & 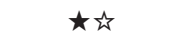 & $\star \star \star$ \\
\hline (35) & 大支方公 & $\star$ 云 & $\star \star \star \star$ \\
\hline
\end{tabular}

Enterococcus faecalis (E. faecalis), Escherichia coli (E. coli), Klebsiella pneumoniae (K. pneumoniae) and Streptococcus gordonii (S. gordonii) ascended in 2 clinical studies $(22,30)$.

The decrease of the phylum Firmicutes was observed after RYGB $(21,24,29)$, as verified in 2 studies after SG $(19,31)$. The abundance of Clostridiales were found decreased in 4 studies $(20,25,32,36)$. The reduced concentration of Clostridiaceae was found in non-obese Wistar rats after RYGB operation (29). Fecal sample of 11 severely obese people who experienced BIB also revealed a reduction of Clostridiaceae (20). An examination of the impact of RYGB on the modifications of gut microbiota in 2013 demonstrated a marked decrease in Blautia (18), and the same result was stated in 2016 by Patrone et al. (20). RYGB (18), SG (36) and BIB (20) could cause a significant reduction in Dorea.

\section{Microbiota altered on moderate evidence}

There is moderate evidence that the population of 20 specific microbial taxa changed after surgery (Table 5 and Supplementary Table 1). The increase of Erysipelotrichales was observed in 2 animal studies $(23,25)$. Three studies found an increase in abundance of Akkermansia (24, 27, 35 ). In 2015, Tremaroli et al. analyzed the population of the genus Citrobacter and Salmonella and the species Enterobacter cancerogenus (E. cancerogenus), Salmonella enterica (S. enterica) and Shigella boydii (S. boydii) (30), and they found an increase after surgery, which was in agreement with a study conducted in 9 individuals in 2013 (26). Two studies showed the increase of Enterobacter after bariatric surgery $(26,30)$. The increased abundance of Parabacteroides was verified by 2 studies on rats $(19,31)$. The abundance of Veillonella was found to 
|L

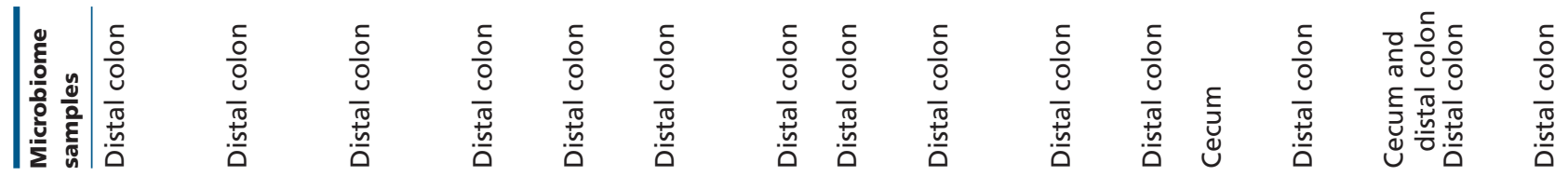

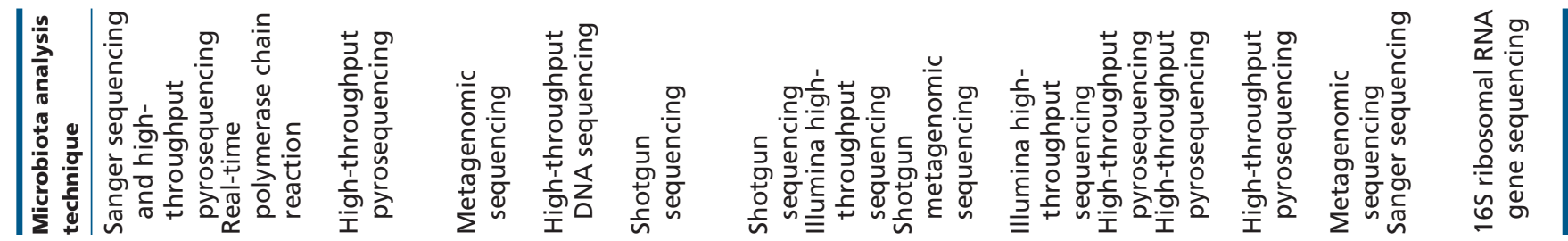

|

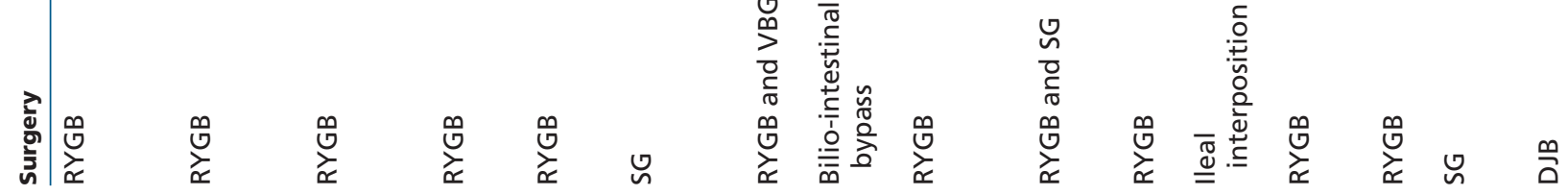

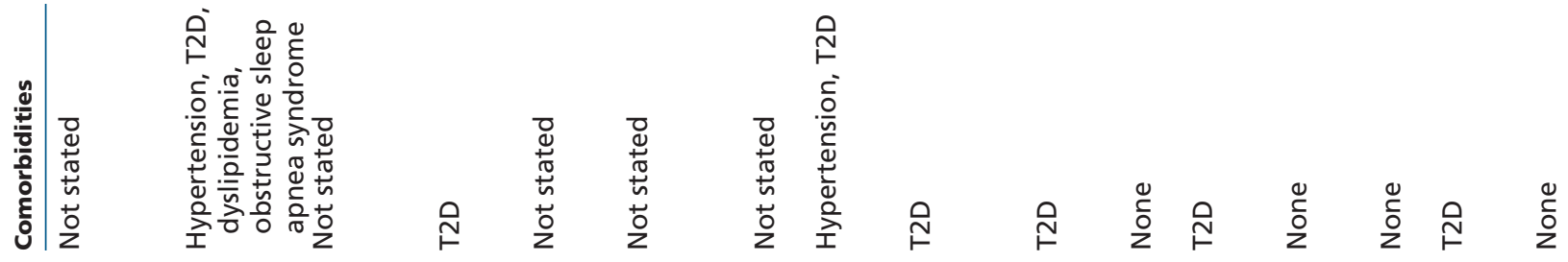

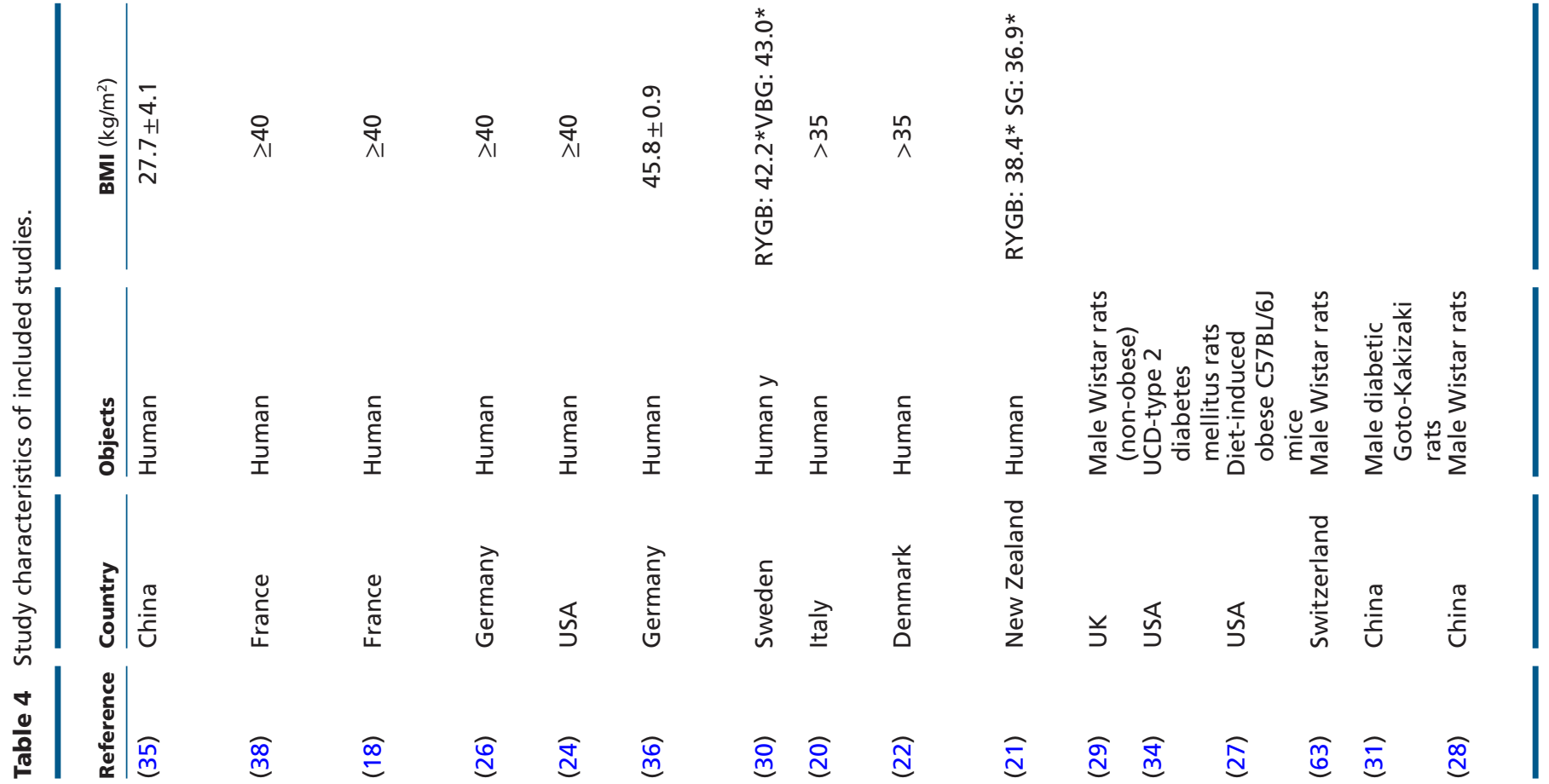



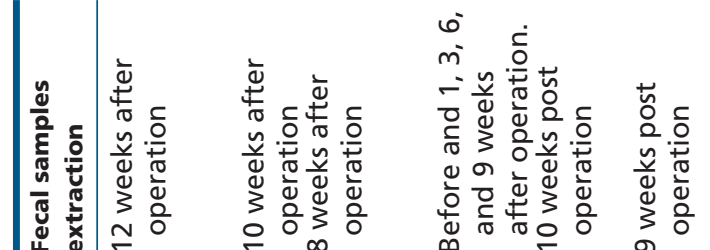

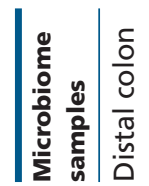

$$
\frac{}{\frac{1}{0}} \frac{\frac{5}{0}}{\frac{0}{0}} \frac{\frac{0}{\pi}}{\frac{\pi}{0}}
$$

$\frac{\frac{5}{0}}{\frac{0}{0}} \frac{\frac{5}{0}}{\frac{0}{\pi}} \frac{\frac{\pi}{\pi}}{\frac{\pi}{0}} \quad \frac{\frac{\pi}{0}}{0}$
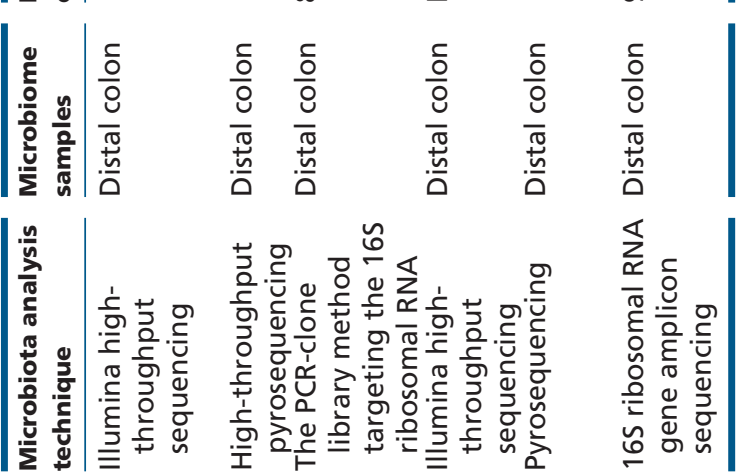

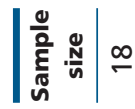

8

6$$
\stackrel{\infty}{\sim}
$$

ㅇำ

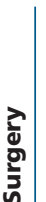

|

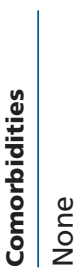

บ

$\stackrel{0}{\check{0}} \stackrel{0}{2}$

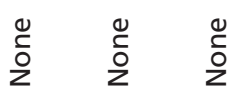

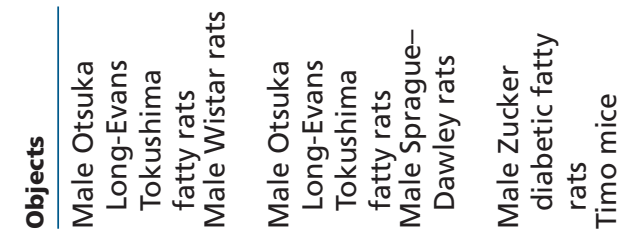

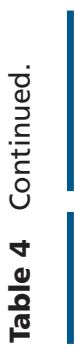

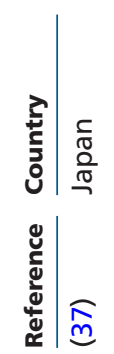

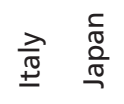

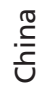

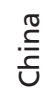
in the population of Coprococcus was found in patients undergoing 3 different bariatric surgeries $(20,26,36)$. The reduction of Faecalibacterium was confirmed in patients undergoing bariatric surgery $(20,26)$. Ruminococcus were found to decrease in 4 studies $(20,23,31,36)$. The decreased abundance of Turicibacter was observed in two studies $(18,37)$.

\section{Microbiota altered on weak and inconclusive evidence}

The population of 51 microbial taxa changed based on a weak level of evidence: the phylum Archaea and Cyanobacteria, class Betaproteobacteria, order Selenomonadales and Verrucomicrobiales, family Bacteroidaceae, Carnobacteriaceae, Coriobacteriaceae, Eubacteriaceae, Lachnospiraceae, Lactobacillaceae, Peptostreptococcaceae and Veillonellaceae and genus Acidaminococcus, Acinetobacter, Aeromonas, Aggregatibacter, Anaerofilum, Campylobacter, Cronobacter, Dermacoccus, Desulfurispirillum, Dickeya, Edwardsiella, Elusimicrobium, Erwinia, Eubacterium, Filifactor, Fusobacterium, Gemmiger, Granulicatella, Klebsiella, Lactococcus, Megasphaera, Neisseria, Oscillibacter, Pantoea, Parasutterella, Pectobacterium, Peptostreptococcus, Phenylobacterium, Proteus, Providencia, Pseudomonas, Rahnella, Serratia, Shewanella, Shigella, Treponema, Vibrio and Yersinia (Table 5 and Supplementary Table 1).

\section{Associations between bacterial population and host metabolic parameters}

The following analysis was mainly based on 9 reports, of which 6 were of good quality $(18,19,20,21,25,38)$ 
Table 5 Level of evidence for alterations of microbiome.

\begin{tabular}{|c|c|c|c|}
\hline & \multicolumn{3}{|c|}{ Levels of evidence } \\
\hline & Strong & Moderate & Weak \\
\hline \multicolumn{4}{|c|}{ Decrease after surgery } \\
\hline Phylum & Firmicutes & & \\
\hline Class & & Clostridia & \\
\hline Order & Clostridiales & & \\
\hline Family & Clostridiaceae & Ruminococcaceae & $\begin{array}{l}\text { Peptostreptococcaceae, Carnobacteriaceae, } \\
\text { Coriobacteriaceae, Eubacteriaceae, } \\
\text { Lachnospiraceae }\end{array}$ \\
\hline Genus & Blautia, Dorea & $\begin{array}{l}\text { Anaerostipes, } \\
\text { Coprococcus, } \\
\text { Faecalibacterium, } \\
\text { Turicibacter, } \\
\text { Clostridium, } \\
\text { Ruminococcus }\end{array}$ & $\begin{array}{l}\text { Eubacterium, Gemmiger, Granulicatella, } \\
\text { Lactococcus, Oscillibacter }\end{array}$ \\
\hline \multicolumn{4}{|l|}{ Species } \\
\hline \multicolumn{4}{|c|}{ Increase after surgery } \\
\hline Phylum & $\begin{array}{l}\text { Bacteroidetes, Fusobacteria, } \\
\text { Proteobacteria, Verrucomicrobia }\end{array}$ & & $\begin{array}{l}\text { Cyanobacteria, Betaproteobacteria, } \\
\text { Selenomonadales, Archaea }\end{array}$ \\
\hline Class & Gammaproteobacteria & & \\
\hline Order & Enterobacteriales, Lactobacillales & Erysipelotrichales & Verrucomicrobiales \\
\hline Family & Enterobacteriaceae & & Bacteroidaceae, Lactobacillaceae, Veillonellaceae \\
\hline Genus & Alistipes, Enterococcus, Escherichia & $\begin{array}{l}\text { Akkermansia, } \\
\text { Citrobacter, } \\
\text { Enterobacter, } \\
\text { Parabacteroides, } \\
\text { Salmonella, } \\
\text { Veillonella }\end{array}$ & $\begin{array}{l}\text { Acidaminococcus, Acinetobacter, Aeromonas, } \\
\text { Aggregatibacter, Anaerofilum, Campylobacter, } \\
\text { Cronobacter, Dermacoccus, Desulfurispirillum, } \\
\text { Dickeya, Edwardsiella, Elusimicrobium, Erwinia, } \\
\text { Filifactor, Fusobacterium, Klebsiella, } \\
\text { Megasphaera, Neisseria, Pantoea, Parasutterella, } \\
\text { Pectobacterium, Peptostreptococcus, } \\
\text { Phenylobacterium, Proteus, Providencia, } \\
\text { Pseudomonas, Rahnella, Serratia, Shewanella, } \\
\text { Shigella, Treponema, Vibrio, Yersinia }\end{array}$ \\
\hline Species & $\begin{array}{l}\text { Enterobacter hormaechei, Enterococcus } \\
\text { faecalis, Escherichia coli, Klebsiella } \\
\text { pneumoniae, Streptococcus gordonii }\end{array}$ & $\begin{array}{l}\text { Enterobacter } \\
\text { cancerogenus, } \\
\text { Salmonella } \\
\text { enterica, Shigella } \\
\text { boydii, Veillonella } \\
\text { dispar, Veillonella } \\
\text { parvula }\end{array}$ & \\
\hline
\end{tabular}

and 3 were of poor quality $(26,28,36)$. Statistical methods, such as Spearman's correlation tests, principal component analysis and univariate linear mixed models were frequently used in relevant studies. Only statistically significant results $(P \leq 0.05)$ were extracted.

\section{Corpulence parameters}

The abundance of Bifidobacterium was positively correlated to body mass index (BMI), body weight, calorie intake and leptin concentration; however, a negative correlation was found with the same parameters for E. coli, observed in patients undergoing RYGB (38). In 2013, the same researchers extended the results by using a pyrosequencing method and found 5 bacterial genera (Bifidobacterium, Blautia, Dorea, Lactobacillus and
Turicibacter) showed positive correlations with most corpulence parameters, and another 8 genera (Bacteroides, Escherichia, Alistipes, Campylobacter, Neisseria, Filifactor, Peptostreptococcus and Gemella) were negatively correlated with most corpulence parameters (18). In the same year, Graessler et al. found that BMI of patients undergoing RYGB correlated to 10 bacterial species, of which Acinetobacter johnsonii (A. johnsonii), Coprococcus comes (C. comes), Faecalibacterium prausnitzii (F. prausnitzii), Fibrobacter succinogenes, Lactobacillus acidophilus, Lactobacillus reuteri, Thermomicrobium roseum ( $T$. roseum) and Treponema pallidum (T. pallidum) were directly associated with BMI (26). Furthermore, E. cancerogenus and $V$. dispar were inversely associated with BMI (26). Moreover, the body weight was found to be negatively correlated with the abundance of Bacteroidetes after SG (36), Treponema after 


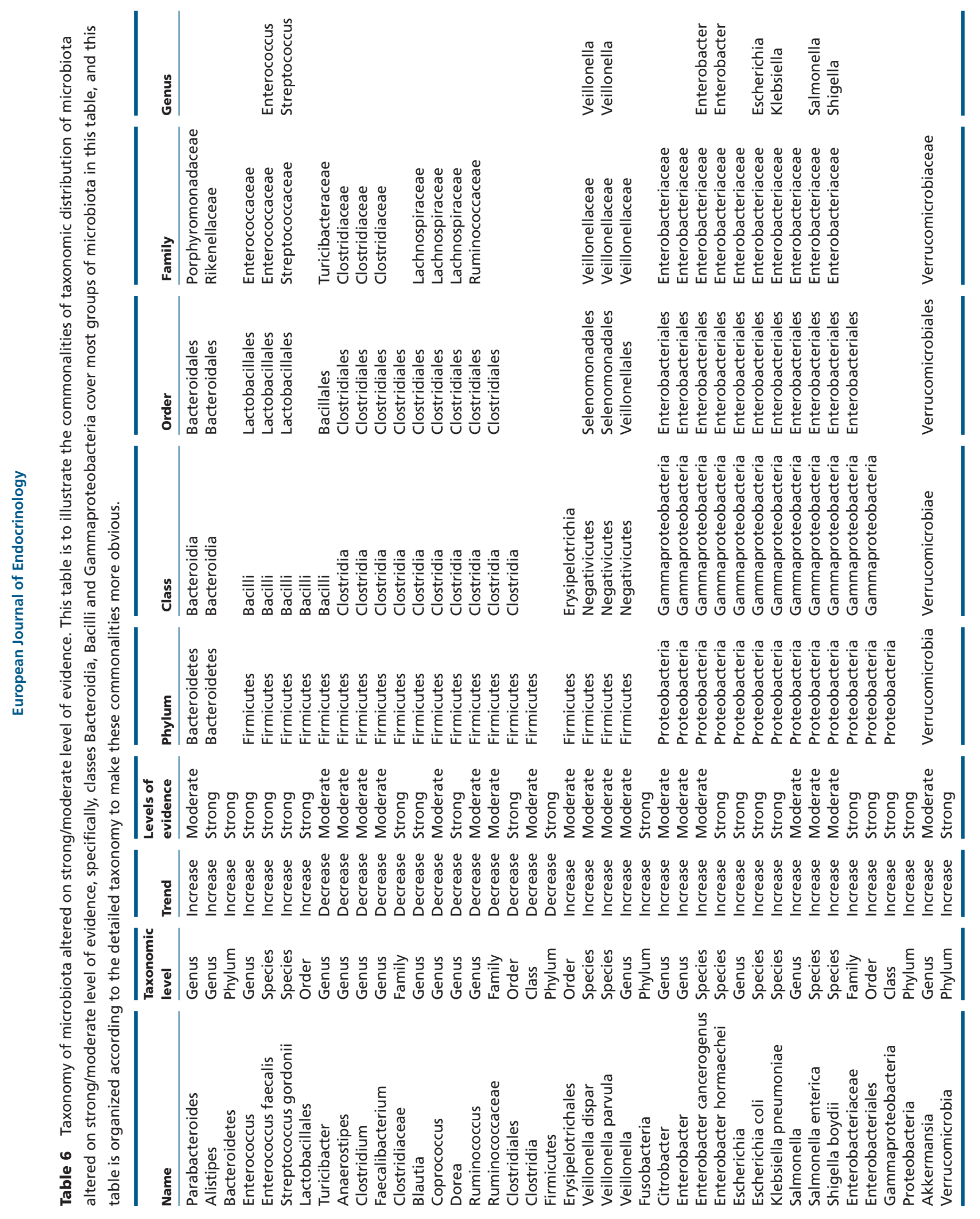


RYGB and SG (19) and Gammaproteobacteria after RYGB and SG (25) and positively correlated with the abundance of the phylum Firmicutes after SG (36).

\section{Glucose homeostasis}

Ling-Chun Kong et al. found that after RYGB, 10 of the 14 discriminating gut microbiota genera were correlated with one or more of the 4 glucose metabolism-related parameters (fasting glycemia, glycated hemoglobin, homeostasis model assessment of insulin resistance (HOMA-IR) and insulinemia) (18). However, a study by Graessler et al. indicated correlations for $F$. prausnitzii, $T$. roseum and $V$. parvula with glucose homeostasis in patients after RYGB (26). A positive connection between the variation of Clostridium and blood insulin was also exhibited in patients undergoing BIB (20). Study on patients receiving RYGB or SG showed a negative correlation for Dialister and Megamonas with HOMA-IR (21). Guo et al. found that the serum insulin in patients undergoing SG and RYGB negatively correlated with the abundance of Alistipes, Anaerofilum, Clostridium, Dorea, Enterococcus, Fusobacterium, Parabacteroides and Veillonella (19).

\section{Lipid profile}

Overall, 6 discriminating gut microbiota genera, Alistipes, Bacteroides, Blautia, Dorea, Escherichia and Phenylobacterium, were associated with cholesterol metabolism, observed in patients undergoing RYGB (18). Furthermore, F. succinogenes, A. johnsonii, T. pallidum, T. roseum, C. spiroforme and Anaerostipes caccae (A. caccae) were significantly positively correlated with several lipid parameters in another study applying RYGB (26); moreover, E. cancerogenus, $V$. parvula, $V$. dispar, and $S$. enterica were negatively correlated with plasma total cholesterol and low-density lipoprotein cholesterol in patients undergoing RYGB (26). A connection between triglyceride and 5 bacterial species (A. johnsonii, A. caccae, E. cancerogenus, $T$. pallidum and $V$. parvula) was also found (26). In addition, an animal study applying RYGB and SG in 2016 showed a negative correlation between total cholesterol and Parasutterella, Treponema and Veillonella (19).

\section{Bile acid}

As for patients receiving SG, a significant direct correlation with lithocholic acid (LCA) was found for F. prausnitzii, whereas plasma deoxycholic acid (DCA) was positively correlated with the abundance of Clostridium sp. L2-50, C. comes and Lachnospiraceae bacterium (36). As for rats undergoing DJB, significant connections have been found that colonic total bile acid was positively correlated with the abundance of Proteobacteria and negatively correlated with the abundance of Bacteroidetes (28).

\section{Highlighted microbiota}

Some specific microbiota showed connections with several different parameters according to more than one study. First, the species $F$. prausnitzii had a positive correlation with BMI and glucose level in patients undergoing RYGB (26), LCA in patients undergoing SG (36) and inflammatory factors such as C-reactive protein (CRP), interleukin-6 and orosomucoid levels in patients receiving RYGB (38). Second, the Lactobacillus and its species Lactobacillus reuteri were found to be positively correlated with BMI in patients undergoing RYGB $(18,26)$. Third, the variation of C. comes was found to be positively correlated with BMI and CRP in patients undergoing RYGB (26) and DCA in patients undergoing SG (36).

\section{Further investigation of the functions of microbiota}

Change of short-chain fatty acids (SCFAs)

By measuring the levels of fecal SCFAs and branched chain fatty acids (BCFAs), it was found that the SCFAs levels were consistent between animals with and without RYGB (27); this was also demonstrated for patients after BIB (20). However, patients undergoing RYGB showed a decrease in the SCFAs/BCFAs ratio (30).

\section{Change of genetic content}

By applying the Kyoto Encyclopedia of Genes and Genomes (KEGG) biological categories, a total of 6 clinical studies assigned the extracted gut genes to different KEGG Othologues (KOs) and annotated the function of the genes and analyzed the metabolic capacity of the gut microbiota through related KEGG modules and KEGG pathways $(18,21,22,26,30,36)$. The phosphotransferase system (PTS) was upregulated after RYGB (26), further proved by 2 human studies $(22,30)$. ABC transporters were enriched after VBG (30) and downregulated after RYGB (18). Nitrogen metabolism, fatty acid metabolism and the two-component system were also upregulated after bariatric surgery (30). Moreover, three KOs related 
to butanoate metabolism, K00074, K00929 and K00626, were significantly higher after SG (36). A total of 53 microbial KEGG modules increased after RYGB, which implied an increased oxygen tolerance and the utilization of macronutrients and micronutrients (22). The increased use of sulfur relay and phosphotransferase systems, purine metabolism and an increase in several nutrient transport systems were observed after RYGB (21).

\section{Transplantation of gut microbiota}

Transplantation of the gut microbiota from bariatric surgery donors to germ-free mice was applied in 2 studies, and the donors were mice and patients, respectively $(27,30)$.The results showed significant weight loss, a lower adiposity index, a lower leptin level and more SCFAs in mice colonized with RYGB microbiota than those in mice colonized with a sham group microbiota (27). Further, a gain of less fat mass was found in mice colonized with RYGB and VGB microbiota than in mice colonized with people with obesity' microbiota (30).

\section{Discussion}

To the best of our knowledge, this is the first systematic review to quantitatively compare the current literature on the gut microbiome of patients or rodents after bariatric surgery. Our study is unique in that it systematically compiles the interaction between the host metabolism and the microbiome. Only with a standardized and strict statistical methodology can the particular correlation of bariatric surgery and the gut microbiome be unbiasedly summarized. The result of this systematic review furthers our understanding of the gut microbiome, which may contribute a great deal to regulating obesity-related conditions after bariatric surgery.

Many previous studies observed an overall lower bacterial diversity and a lower relative abundance of Bacteriodetes in individuals with obesity $(39,40)$. Although controversial results were found regarding the change of richness and diversity of microbiota related to the bariatric surgery procedures, four studies had reached an agreement on the higher gut microbiota diversity, as estimated by the Shannon index after surgery $(19,20,22,23)$. This fact revealed that the surgery itself or the metabolic benefit conferred by surgery allowed the modulation of the gut microbiota. Kong et al. (18) suggested that the change in eating habits might account for this change in microbiota diversity, such as reducing the amount of fat and increasing the consumption of polysaccharides (41). However, in our previous study (19), the pair-fed group served as the RYGB diet-matched control group. Thus, the increased microbiota diversity induced by RYGB may be food independent.

Based on strong evidence, the population of Proteobacteria and its class Gammaproteobacteria, order Enterobacteriales, family Enterobacteriaceae and genus Escherichia increases after bariatric surgery. Several genera (Citrobacter, Enterobacter, Escherichia and Salmonella) and species (E. cancerogenus, E. hormaechei, E. coli, K. pneumoniae and S. enterica) belong to the family Enterobacteriaceae also increase on strong or moderate evidence (Table 6). Mice with obesity and insulin resistance induced by a high-fat diet had improved insulin sensitivity concomitant with a significant overrepresentation of the phylum Proteobacteria when given the antibiotics ampicillin, neomycin, and metronidazole by mouth, which showed that the abundance of the phylum Proteobacteria and insulin sensitivity may be linked (42).

Within the order Clostridiales (an abundant order of the phylum Firmicutes), there are 7 genera (Anaerostipes, Clostridium, Faecalibacterium, Blautia, Coprococcus, Dorea and Ruminococcus) and 2 families (Clostridiaceae and Ruminococcaceae) that decreased after surgery on strong or moderate evidence (Table 6). This is to be expected because several reports found the decline of Clostridium in the fecal samples from the gut of people with obesity $(20,31,37)$. Because the production of butyrate appears to be a underlying feature of a few bacterial taxa within the order Clostridiales, a significant change of the population of such bacteria furthers the high interest on the crosstalk between them and host metabolism (43, 44, 45, 46). Additionally, the anaerobic gram-positive bacterium Clostridium difficile causes intestinal infections responsible for symptoms ranging from mild diarrhea to fulminant colitis (47).

F. prausnitzii, the sole known species of the genus Faecalibacterium, seemed to be of great importance in the inflammatory process and glucose homeostasis, as demonstrated by 3 studies $(26,36,38)$. F . prausnitzii is an anti-inflammatory commensal bacterium that was identified by gut microbiota analysis (48), which is also found at lower abundance in those with Crohn's disease (49).

Lactobacillus contributed more to body composition, and two studies indicated correlations for the abundance of Lactobacillus and BMI $(18,26)$. Lactobacillus can play a role by producing lactic acid and extracting energy 
from the fermentation of different sugars, which helps regulate the corpulence metabolism in people with obesity (50). Because bariatric surgery delays glucose and amino absorption in the host tract, the increase in simple sugars and amino acids in the large intestine may activate the colonic bacteria to derive energy from malabsorbed nutrients $(51,52,53)$.

Although C. comes has been found to be correlated with the inflammation procedure and bile acid homeostasis, the mechanism that affects the host metabolism remains unclear, which suggests more investigations $(54,55,56)$. Previous studies showed that agglutinating antibodies to $C$. comes are more frequently found in the sera of Crohn's patients than that in ulcerative colitis patients and healthy subjects, which suggests its important role in patients with Crohn's disease $(54,56,57)$.

Approximately 5-10\% of bile acids are biotransformed and degraded by intestinal bacteria during the enterohepatic circulation (58). The alteration in bile flow after RYGB may have considerable effects on the composition of the gut microbiota. Changes to the gut microbiome are also seen following SG, this is supported from studies interfering with BA homeostasis and the gut microbiome in a bidirectional way (59). Therefore, it is likely the gut microbiota mediates the phenotype and its metabolic complications in part through bile acid co-metabolism. More studies are needed to discover the relationship between bile acid and gut microbiota (60).

Previous studies have shown the difference between obese microbiota and lean microbiota. Obese microbiota may produce more SCFAs, which impede glucose incorporation in adipocytes and modulates energy expenditure through the sympathetic neuronal network. Thus, more energy can be extracted from a certain diet $(61,62)$. However, the results of our study failed to converge to a common conclusion regarding the change of SCFAs after bariatric surgery.

Due to the limited number of included studies and the different methodologies and technique applied in studies on microbiota following bariatric surgery, inconsistent findings were inevitable. Although in this review, we used the evidence level system to analyze and summarize the existing literature, there is still a justified need in the field to elucidate useful information that could move this line of research forward. This review could serve as an incentive for the field to harmonize techniques and improve the study design to generate complementary findings. More advanced sequencing technology and metagenomic and bioinformatic methods are needed to investigate the microorganisms inhabiting the human gut. As for the type of design, a non-surgical weight loss treatment group for control in the human study and a pair-fed and weightlimited control group in the animal study are needed for further research. However, randomized and double-blind studies are currently extremely scarce in this field.

\section{Limitation}

Our study has some limitations. First, the low cohort numbers may influence the results. Second, the different diet and comorbidities may influence the gut microbiome. Third, the time point to extract the fecal sample are inconsistent among studies, however, the diet after bariatric surgery may change a lot with time going, so the gut microbiome may be affected. Fourth, the difference on varying types of surgery remains unclear according to our results.

\section{Conclusion}

Bariatric surgery is included in the treatment of $\mathrm{T} 2 \mathrm{D}$ and obesity, and gut microbiota were implicated in the crosstalk of host metabolic change and bariatric surgery. In this review, we found that microbial composition is greatly altered after bariatric surgery. Based on strong evidence, 4 phyla (Bacteroidetes, Fusobacteria, Verrucomicrobia and Proteobacteria) increased after surgery; within the phylum Firmicutes, Lactobacillales, Enterococcus, E. faecalis and S. gordonii increased; and within the phylum Proteobacteria, Gammaproteobacteria, Enterobacteriales, Enterobacteriaceae, Escherichia, Alistipes, E. coli, E. hormaechei and K. pneumoniae increased. The decreased microbial groups were Firmicutes, Clostridiales, Clostridiaceae, Blautia and Dorea. Lactobacillus, F. prausnitzii and C. comes are more related to the metabolic alteration of patients after bariatric surgery. The results of gut genetic content, microbiome transplantation and SCFAs production imply the potential of microbiota in modulating host metabolic regulation. More long-term prospective studies are warranted to investigate the mechanism of gut microbiota on modulating host metabolism after bariatric surgery.

Supplementary data

This is linked to the online version of the paper at https://doi.org/10.1530/ EJE-17-0403. 
Declaration of interest

The authors declare that there is no conflict of interest that could be perceived as prejudicing the impartiality of the case study reported.

\section{Funding}

This study was supported by the National Natural Science Foundation of China award No. 81300684 and 81700745.

\section{Author contribution statement}

Yuan Sheng and Da-Jin Zou designed the research study; Yan Guo, Zhi-Ping Huang and Chao-Qian Liu performed the research; Yan Guo, Zhi-Ping Huang and Chao-Qian Liu analyzed the data; Yan Guo, Zhi-Ping Huang wrote the paper; Yuan Sheng, Da-Jin Zou and Lin Qi revised the paper.

\section{References}

1 Sjostrom L, Lindroos AK, Peltonen M, Torgerson J, Bouchard C, Carlsson B, Dahlgren S, Larsson B, Narbro K, Sjostrom CD et al. Lifestyle, diabetes, and cardiovascular risk factors 10 years after bariatric surgery. New England Journal of Medicine 2004351 2683-2693. (https://doi.org/10.1056/NEJMoa035622)

2 Rubino F, Nathan DM, Eckel RH, Schauer PR, Alberti KGMM, Zimmet PZ, Del Prato S, Ji L, Sadikot SM, Herman WH et al. Metabolic surgery in the treatment algorithm for type 2 diabetes: a joint statement by international diabetes organizations. Diabetes Care 201639 861-877. (https://doi.org/10.2337/dc16-0236)

3 Buchwald H, Estok R, Fahrbach K, Banel D, Jensen MD, Pories WJ, Bantle JP \& Sledge I. Weight and type 2 diabetes after bariatric surgery: systematic review and meta-analysis. American Journal of Medicine 2009122 248.e245-256.e245. (https://doi.org/10.1016/ j.amjmed.2008.12.006)

4 Aron-Wisnewsky J, Dore J \& Clement K. The importance of the gut microbiota after bariatric surgery. Nature Reviews Gastroenterology and Hepatology 20129 590-598. (https://doi.org/10.1038/ nrgastro.2012.161)

5 Burcelin R, Serino M, Chabo C, Garidou L, Pomie C, Courtney M, Amar J \& Bouloumie A. Metagenome and metabolism: the tissue microbiota hypothesis. Diabetes, Obesity and Metabolism 201315 61-70. (https://doi.org/10.1111/dom.12157)

6 Arora T \& Bäckhed F. The gut microbiota and metabolic disease: current understanding and future perspectives. Journal of Internal Medicine $2016280339-349$

7 Magouliotis DE, Tasiopoulou VS, Sioka E, Chatedaki C \& Zacharoulis D. Impact of bariatric surgery on metabolic and gut microbiota profile: a systematic review and meta-analysis. Obesity Surgery 201727 1345-1357.

8 Strader AD. Ileal transposition provides insight into the effectiveness of gastric bypass surgery. Physiology and Behavior 200688 277-282. (https://doi.org/10.1016/j.physbeh.2006.05.034)

9 Moher D, Liberati A, Tetzlaff J \& Altman DG. Preferred reporting items for systematic reviews and meta-analyses: the PRISMA statement. BMJ 2009339 b2535. (https://doi.org/10.1136/bmj. b2535)

10 Zhou Y, Gao J, Luo L \& Wang Y. Does bruxism contribute to dental implant failure? A systematic review and meta-analysis. Clinical Implant Dentistry and Related Research 201618 410-420. (https://doi. org/10.1111/cid.12300)

11 Zhu H, Shen Z, Luo H, Zhang W \& Zhu X. Chlamydia trachomatis infection-associated risk of cervical cancer: a metaanalysis. Medicine 201695 e3077. (https://doi.org/10.1097/ MD.0000000000003077)
12 Kilkenny C, Browne WJ, Cuthill IC, Emerson M \& Altman DG. Improving bioscience research reporting: the ARRIVE guidelines for reporting animal research. PLoS Biology 20108 e1000412. (https://doi.org/10.1371/journal.pbio.1000412)

13 Scholten-Peeters GGM, Verhagen AP, Bekkering GE, van der Windt DAWM, Barnsley L, Oostendorp RAB \& Hendriks EJM. Prognostic factors of whiplash-associated disorders: a systematic review of prospective cohort studies. Pain 2003104 303-322. (https://doi.org/10.1016/S0304-3959(03)00050-2)

14 Kuijpers T, van der Windt DA, van der Heijden GJ \& Bouter LM. Systematic review of prognostic cohort studies on shoulder disorders. Pain 2004109 420-431. (https://doi.org/10.1016/j.pain.2004.02.017)

15 Mols F, Vingerhoets AJ, Coebergh JW \& van de Poll-Franse LV. Quality of life among long-term breast cancer survivors: a systematic review. European Journal of Cancer 200541 2613-2619. (https://doi. org/10.1016/j.ejca.2005.05.017)

16 Oczkowski SJ, Chung HO, Hanvey L, Mbuagbaw L \& You JJ. Communication tools for end-of-life decision-making in the intensive care unit: a systematic review and meta-analysis. Critical Care 201620 97. (https://doi.org/10.1186/s13054-016-1264-y)

17 Chao A. Nonparametric estimation of the number of classes in a population. Scandinavian Journal of Statistics 198411 265-270.

18 Kong LC, Tap J, Aron-Wisnewsky J, Pelloux V, Basdevant A, Bouillot JL, Zucker JD, Dore J \& Clement K. Gut microbiota after gastric bypass in human obesity: increased richness and associations of bacterial genera with adipose tissue genes. American Journal of Clinical Nutrition 201398 16-24. (https://doi.org/10.3945/ ajcn.113.058743)

19 Guo Y, Liu CQ, Shan CX, Chen Y, Li HH, Huang ZP \& Zou DJ. Gut microbiota after Roux-en-Y gastric bypass and sleeve gastrectomy in a diabetic rat model: increased diversity and associations of discriminant genera with metabolic changes. Diabetes/Metabolism Research and Reviews 201633 1-8. (https://doi.org/10.1002/ dmrr.2857)

20 Patrone V, Vajana E, Minuti A, Callegari ML, Federico A, Loguercio C, Dallio M, Tolone S, Docimo L \& Morelli L. Postoperative changes in fecal bacterial communities and fermentation products in obese patients undergoing bilio-intestinal bypass. Frontiers in Microbiology 20167200.

21 Murphy R, Tsai P, Jullig M, Liu A, Plank L \& Booth M. Differential changes in gut microbiota after gastric bypass and sleeve gastrectomy bariatric surgery vary according to diabetes remission. Obesity Surgery 201627 917-925.

22 Palleja A, Kashani A, Allin KH, Nielsen T, Zhang C, Li Y, Brach T, Liang S, Feng Q, Jorgensen NB et al. Roux-en-Y gastric bypass surgery of morbidly obese patients induces swift and persistent changes of the individual gut microbiota. Genome Medicine 2016867. (https://doi.org/10.1186/s13073-016-0312-1)

23 Basso N, Soricelli E, Castagneto-Gissey L, Casella G, Albanese D, Fava F, Donati C, Tuohy K, Angelini G, La Neve F et al. Insulin resistance, microbiota and fat distribution changes by a new model of vertical sleeve gastrectomy in obese rats. Diabetes $2016 \mathbf{6 1}$ 2990-3001. (https://doi.org/10.2337/db16-0039)

24 Ward EK, Schuster DP, Stowers KH, Royse AK, Ir D, Robertson CE, Frank DN \& Austin GL. The effect of PPI use on human gut microbiota and weight loss in patients undergoing laparoscopic Roux-en-Y gastric bypass. Obesity Surgery 201424 1567-1571. (https://doi.org/10.1007/s11695-014-1275-1)

25 Shao Y, Ding R, Xu B, Hua R, Shen Q, He K \& Yao Q. Alterations of gut microbiota after roux-en-Y gastric bypass and sleeve gastrectomy in Sprague-Dawley rats. Obesity Surgery 201627 295-302.

26 Graessler J, Qin Y, Zhong H, Zhang J, Licinio J, Wong ML, Xu A, Chavakis T, Bornstein AB, Ehrhart-Bornstein $\mathrm{M}$ et al. Metagenomic sequencing of the human gut microbiome before and after bariatric surgery in obese patients with type 2 diabetes: correlation with inflammatory and metabolic parameters. Pharmacogenomics Journal 201313 514-522. (https://doi.org/10.1038/tpj.2012.43) 
27 Liou AP, Paziuk M, Luevano JM Jr, Machineni S, Turnbaugh PJ \& Kaplan LM. Conserved shifts in the gut microbiota due to gastric bypass reduce host weight and adiposity. Science Translational Medicine 20135 178ra141.

28 Zhang X, Wang Y, Zhong M, Liu T, Han H, Zhang G, Liu S, Wei M, Wu Q \& Hu S. Duodenal-jejunal bypass preferentially elevates serum taurine-conjugated bile acids and alters gut microbiota in a diabetic rat model. Obesity Surgery 201526 1890-1899.

29 Li JV, Ashrafian H, Bueter M, Kinross J, Sands C, le Roux CW, Bloom SR, Darzi A, Athanasiou T, Marchesi JR et al. Metabolic surgery profoundly influences gut microbial-host metabolic cross-talk. Gut 201160 1214-1223. (https://doi.org/10.1136/gut.2010.234708)

30 Tremaroli V, Karlsson F, Werling M, Stahlman M, KovatchevaDatchary P, Olbers T, Fandriks L, Le Roux CW, Nielsen J \& Backhed F. Roux-en-Y gastric bypass and vertical banded gastroplasty induce long-term changes on the human gut microbiome contributing to fat mass regulation. Cell Metabolism 201522 228-238. (https://doi org/10.1016/j.cmet.2015.07.009)

31 Huang X, Weng P, Zhang H \& Lu Y. Remodeling intestinal flora with sleeve gastrectomy in diabetic rats. Journal of Diabetes Research 2014 2014196312.

32 Jiang S, Wang Q, Huang Z, Song A, Peng Y, Hou S, Guo S, Zhu W, Yan S, Lin Z et al. Gastric bypass surgery reverses diabetic phenotypes in Bdnf-deficient mice. American Journal of Pathology 2016186 2117-2128. (https://doi.org/10.1016/j.ajpath.2016.04.009)

33 Kashihara H, Shimada M, Yoshikawa K, Higashijima J, Nakao T, Nishi M \& Takasu C. Duodenal-jejunal bypass changes the composition of the gut microbiota. Surgery Today 201647 137-140.

34 Cummings BP, Bettaieb A, Graham JL, Kim J, Ma F, Shibata N, Stanhope KL, Giulivi C, Hansen F, Jelsing J et al. Bile-acid-mediated decrease in endoplasmic reticulum stress: a potential contributor to the metabolic benefits of ileal interposition surgery in UCD-T2DM rats. Disease Models and Mechanisms 20136 443-456. (https://doi. org/10.1242/dmm.010421)

35 Zhang H, DiBaise JK, Zuccolo A, Kudrna D, Braidotti M, Yu Y, Parameswaran P, Crowell MD, Wing R, Rittmann BE et al. Human gut microbiota in obesity and after gastric bypass. PNAS $2009 \mathbf{1 0 6}$ 2365-2370. (https://doi.org/10.1073/pnas.0812600106)

36 Damms-Machado A, Mitra S, Schollenberger AE, Kramer KM, Meile T, Konigsrainer A, Huson DH \& Bischoff SC. Effects of surgical and dietary weight loss therapy for obesity on gut microbiota composition and nutrient absorption. BioMed Research International 20152015806248.

37 Miyachi T, Nagao M, Shibata C, Kitahara Y, Tanaka N, Watanabe K, Tsuchiya T, Motoi F, Naitoh T \& Unno M. Biliopancreatic limb plays an important role in metabolic improvement after duodenaljejunal bypass in a rat model of diabetes. Surgery 2016159 1360-1371.

38 Furet JP, Kong LC, Tap J, Poitou C, Basdevant A, Bouillot JL, Mariat D, Corthier G, Dore J, Henegar C et al. Differential adaptation of human gut microbiota to bariatric surgery-induced weight loss: links with metabolic and low-grade inflammation markers. Diabetes 201059 3049-3057. (https://doi.org/10.2337/db10-0253)

39 Turnbaugh PJ, Hamady M, Yatsunenko T, Cantarel BL, Duncan A, Ley RE, Sogin ML, Jones WJ, Roe BA, Affourtit JP et al. A core gut microbiome in obese and lean twins. Nature $2009 \mathbf{4 5 7} 480-484$. (https://doi.org/10.1038/nature07540)

40 Turnbaugh PJ, Backhed F, Fulton L \& Gordon JI. Diet-induced obesity is linked to marked but reversible alterations in the mouse distal gut microbiome. Cell Host and Microbe 20083 213-223. (https://doi. org/10.1016/j.chom.2008.02.015)

41 le Roux CW, Bueter M, Theis N, Werling M, Ashrafian H, Lowenstein C, Athanasiou T, Bloom SR, Spector AC, Olbers T et al. Gastric bypass reduces fat intake and preference. American Journal of
Physiology: Regulatory, Integrative and Comparative Physiology 2011301 R1057-R1066. (https://doi.org/10.1152/ajpregu.00139.2011)

42 Carvalho BM, Guadagnini D, Tsukumo DM, Schenka AA, LatufFilho P, Vassallo J, Dias JC, Kubota LT, Carvalheira JB \& Saad MJ. Modulation of gut microbiota by antibiotics improves insulin signalling in high-fat fed mice. Diabetologia 201255 2823-2834. (https://doi.org/10.1007/s00125-012-2648-4)

43 Mitchell WJ. The phosphotransferase system in solventogenic clostridia. Journal of Molecular Microbiology and Biotechnology 201525 129-142. (https://doi.org/10.1159/000375125)

44 Kucerova P \& Cervinkova M. Spontaneous regression of tumour and the role of microbial infection-possibilities for cancer treatment. Anticancer Drugs 201627 269-277. (https://doi.org/10.1097/ CAD.0000000000000337)

45 Stock I. Tetanus and Clostridium tetani-a brief review. Medizinische Monatsschrift für Pharmazeuten 201538 57-60.

46 Omernik A \& Plusa T. Toxins of Clostridium perfringens as a natural and bioterroristic threats. Polski Merkuriusz Lekarski 201539 149-152.

47 Bordeleau E \& Burrus V. Cyclic-di-GMP signaling in the gram-positive pathogen Clostridium difficile. Current Genetics 201561 497-502. (https://doi.org/10.1007/s00294-015-0484-z)

48 Sokol H, Pigneur B, Watterlot L, Lakhdari O, Bermudez-Humaran LG, Gratadoux JJ, Blugeon S, Bridonneau C, Furet JP, Corthier G et al. Faecalibacterium prausnitzii is an anti-inflammatory commensal bacterium identified by gut microbiota analysis of Crohn disease patients. PNAS 2008105 16731-16736. (https://doi.org/10.1073/ pnas.0804812105)

49 Wright EK, Kamm MA, Teo SM, Inouye M, Wagner J \& Kirkwood CD. Recent advances in characterizing the gastrointestinal microbiome in Crohn's disease: a systematic review. Inflammatory Bowel Diseases 201521 1219-1228.

50 Scheepers LE, Penders J, Mbakwa CA, Thijs C, Mommers M \& Arts IC. The intestinal microbiota composition and weight development in children: the KOALA Birth Cohort Study. International Journal of Obesity 201539 16-25. (https://doi.org/10.1038/ijo.2014.178)

51 Chen CY, Chen SW \& Wang HT. Effect of supplementation of yeast with bacteriocin and Lactobacillus culture on growth performance, cecal fermentation, microbiota composition, and blood characteristics in broiler chickens. Asian-Australasian Journal of Animal Sciences 201630 211-220.

52 Park K, Mera PE, Escalante-Semerena JC \& Brunold TC. Resonance Raman spectroscopic study of the interaction between Co(II)rrinoids and the ATP: corrinoid adenosyltransferase PduO from Lactobacillus reuteri. Journal of Biological Inorganic Chemistry 201621 669-681.

53 Urmann K, Arshavsky-Graham S, Walter JG, Scheper T \& Segal E. Whole-cell detection of live lactobacillus acidophilus on aptamerdecorated porous silicon biosensors. Analyst $20161415432-5440$.

54 Hazenberg MP, van de Merwe JP, Pena AS, Pennock-Schroder AM $\&$ van Lieshout LM. Antibodies to Coprococcus comes in sera of patients with Crohn's disease. Isolation and purification of the agglutinating antigen tested with an ELISA technique. Journal of Clinical and Laboratory Immunology 198723 143-148.

55 Van de Merwe JP \& Stegeman JH. Binding of Coprococcus comes to the Fc portion of IgG. A possible role in the pathogenesis of Crohn's disease? European Journal of Immunology 198515 860-863. (https:// doi.org/10.1002/eji.1830150823)

56 Hazenberg MP, Pennock-Schroder AM \& van de Merwe JP. Culturing and extraction of Coprococcus comes, absorption of serumagglutinins by soluble fractions and relation between agglutinins and antibodies in sera of patients with Crohn's disease. Medical Microbiology and Immunology 1986175 299-306. (https://doi. org/10.1007/BF02126051)

57 Severijnen AJ, Hazenberg MP \& van de Merwe JP. Induction of chronic arthritis in rats by cell wall fragments of anaerobic coccoid 
rods isolated from the faecal flora of patients with Crohn's disease. Digestion 198839 118-125. (https://doi.org/10.1159/000199614)

58 Penney NC, Kinross J, Newton RC \& Purkayastha S. The role of bile acids in reducing the metabolic complications of obesity after bariatric surgery: a systematic review. International Journal of Obesity 201539 1565-1574. (https://doi.org/10.1038/ijo.2015.115)

59 Islam KB, Fukiya S, Hagio M, Fujii N, Ishizuka S, Ooka T, Ogura Y, Hayashi T \& Yokota A. Bile acid is a host factor that regulates the composition of the cecal microbiota in rats. Gastroenterology 2011 141 1773-1781. (https://doi.org/10.1053/j.gastro.2011.07.046)

60 Le CE, Nielsen T, Qin J, Prifti E, Hildebrand F, Falony G, Almeida M, Arumugam M, Batto JM \& Kennedy S. Richness of human gut microbiome correlates with metabolic markers. Nature 2013500 541-546. (https://doi.org/10.1038/nature12506)

61 Albaugh VL, Flynn CR, Tamboli RA \& Abumrad NN. Recent advances in metabolic and bariatric surgery. F1000Research 20165.

62 Oh TJ, Ahn CH \& Cho YM. Contribution of the distal small intestine to metabolic improvement after bariatric/metabolic surgery: lessons from ileal transposition surgery. Journal of Diabetes Investigation 2016 7 94-101. (https://doi.org/10.1111/jdi.12444)

63 Osto M, Abegg K \& Bueter Mle Roux CWCani PDLutz TA Roux-en-Y gastric bypass surgery in rats alters gut microbiota profile along the intestine. Physiology \& Behavior 2013119 92-96. (https://doi. org/10.1016/j.physbeh.2013.06.008)

Received 17 May 2017

Revised version received 14 August 2017

Accepted 15 September 2017 